\title{
Application of GM(1,1) Model in Prediction of Amine Solution Foaming
}

\author{
Fangfang Wang \\ Personnel Department of Sichuan University of Arts and Science, Dazhou, China \\ Email:2867195667@qq.com
}

How to cite this paper: Wang, F.F. (2020) Application of $\operatorname{GM}(1,1)$ Model in Prediction of Amine Solution Foaming. Open Access Library Journal, 7: e5987.

https://doi.org/10.4236/oalib.1105987

Received: December 9, 2019

Accepted: April 24, 2020

Published: April 27, 2020

Copyright $\odot 2020$ by author(s) and Open Access Library Inc.

This work is licensed under the Creative Commons Attribution International License (CC BY 4.0).

http://creativecommons.org/licenses/by/4.0/

\begin{abstract}
Amine solution foaming is affected by many factors, effectively predicting and analyzing the foaming of amine solution, which is of great significance to the smooth operation of the plant and the improvement of product gas quality. Based on the production data of Puguang gas field, the author predicted the foaming condition of amine solution by establishing the mathematical model of $\operatorname{GM}(1,1)$. The results show that the method is simple and the prediction accuracy is relatively high, which can meet the actual production needs.
\end{abstract}

\section{Subject Areas}

Mathematical Analysis

\section{Keywords}

GM(1,1) Model, Foaming, Prediction

\section{Introduction}

Puguang Gas Field is the first high-sulfur gas field in China, its feed gas produced is processed by five working procedures of desulfurization, dehydration, sulfur recovery, exhaust absorption and acid water stripping, and output qualified gas products and high-quality sulfur. The desulfurization unit is the first pass for processing the feed gas. Going through the reverse absorption reaction of the amine solution and absorber, the hydrogen sulfide in the feed gas is removed. Due to macro and micro factors, the foaming phenomenon of the amine solution often occurs, which leads to problems such as the excession of $\mathrm{H}_{2} \mathrm{~S}$ in gas products, worsening environmental pollution, and the decrease of processing capacity equipment $[1]$. 
At present, most domestic scholars' research on amine solution foaming focuses on the analysis and discussion of influencing factors. Guo Linchao et al. [2] [3] [4] combined the actual operation of equipment and their working experience to analyze the factors influencing amine solution foaming and its corresponding response from a macroscopic perspective. However, few studies have been conducted on the prediction of amine solution foaming. Therefore, with the help of the prediction method and the actual operation of the Puguang gas field, the author proposes to establish a $\operatorname{GM}(1,1)$ model to predict the foaming of the amine solution, and to provide a theoretical basis for the actual production.

\section{Preliminary Knowledge}

\subsection{Basic Principles of Grey GM(1,1) Model}

The grey system GM $(1,1)$ model is a widely used dynamic grey prediction model composed of First order differential equation with one variable [5]. In the application process, there is no special requirement for the amount of data, the law of change, etc. The raw data is mainly processed to generate a sequence of numbers with exponential variation, a differential equation is established, and the process described by approximating the time series is fitted to achieve the purpose of data prediction.

\subsection{Modeling Steps}

1) Data prejudgment

Before the model is established, the original data is first checked to ensure its feasibility.

Assume that the original data sequence is represented as $x^{(0)}=\left(x^{(0)}(1), x^{(0)}(2), \cdots, x^{(0)}(n)\right)$, then calculate the scale comparison ratio.

$$
\lambda(k)=\frac{x^{(0)}(k-1)}{x^{(0)}(k)}, k=2,3, \cdots, n
$$

To determine if $\lambda(k)$ falls into the range of $\left(\mathrm{e}^{\frac{-2}{n+1}}, \mathrm{e}^{\frac{2}{n+1}}\right)$, then the $\operatorname{GM}(1,1)$ model can be established for the series $x^{(0)}$.

2) Accumulation process

Accumulate raw data to generate new sequences

$$
x^{(1)}=\left(x^{(1)}(1), x^{(1)}(2), \cdots, x^{(1)}(n)\right)
$$

Among them: $x^{(1)}(k)=\sum_{i=0}^{k} x^{(0)}(i), k=1,2, \cdots, n$

The corresponding differential equation is

$$
\frac{\mathrm{d} x^{(1)}}{\mathrm{d} t}+a x^{(1)}=u
$$

In it, $x^{(1)}$ is a series of numbers obtained after accumulation. $t$ means time; $a$, $u$ are the parameters to be estimated. 
3) Calculate the parameters to be estimated

Use the least squares method to find a,u.

Assume,

$$
B=\left[\begin{array}{cc}
-\frac{1}{2}\left[x^{(1)}(1)+x^{(1)}(2)\right] & 1 \\
-\frac{1}{2}\left[x^{(1)}(2)+x^{(1)}(3)\right] & 1 \\
\vdots & \vdots \\
-\frac{1}{2}\left[x^{(1)}(n-1)+x^{(1)}(n)\right] & 1
\end{array}\right], \quad y_{n}=\left(x^{(0)}(2), x^{(0)}(3), \cdots, x^{(0)}(n)\right)^{\mathrm{T}}
$$

Then

$$
\left[\begin{array}{l}
a \\
u
\end{array}\right]=\left(B^{\mathrm{T}} B\right)^{-1} B^{\mathrm{T}} y_{n}
$$

4) Model solving

Solving the original differential equation, the approximate solution is

$$
x^{(1)}(i+1)=\left[x^{(0)}(i)-\frac{u}{a}\right] \mathrm{e}^{-a i}+\frac{u}{a}
$$

where: $x^{(1)}(1)=x^{(0)}(1), \varepsilon_{i}=x^{(0)}(i)-x^{(2)}(i):$ is the sum of the predicted values.

5) Model accuracy test

Average relative error calculation

$$
\Delta=\frac{1}{n} \sum_{i=1}^{n} \Delta_{i}
$$

where: $\Delta_{i}=\left|\frac{\varepsilon_{i}}{x^{(0)}(i)}\right|, \quad \varepsilon_{i}=x^{(0)}(i)-x^{(2)}(i)$.

6) Mean variance ratio calculation

The ratio calculation formula is:

$$
c=\frac{s_{1}}{s_{0}}
$$

Among them: $s_{0}^{2}$ is the mean variance of the original sequence, and $s_{1}^{2}$ is the Mean variance of residual series.

7) Precision test

Calculate the average relative error and the mean variance ratio, and compare with the precision analysis table (see Table 1) to obtain the precision level of the model.

Table 1. Precision analysis table.

\begin{tabular}{ccc}
\hline Average relative error & Mean variance ratio & Precision level \\
\hline 0.01 & 0.35 & first level \\
0.05 & 0.5 & second level \\
0.1 & 0.65 & three level \\
0.2 & 0.8 & four level \\
$>0.2$ & $>0.8$ & five level \\
\hline
\end{tabular}


8) Model prediction

If the above test meets the requirements, the model can then be used for prediction. The specific prediction formula is as follows:

$$
x^{(1)}(i+1)=\left[x^{(0)}(i)-\frac{u}{a}\right] \mathrm{e}^{-a i}+\frac{u}{a}
$$

Among them:

$$
x^{(2)}(i+1)=x^{(1)}(i+1)-x^{(1)}(i), x^{(2)}(i+2)=x^{(1)}(i+2)-x^{(1)}(i+1)
$$

\section{Example Analysis}

Puguang gas field as an example, during the foaming of the amine solution, the raw data of 10 amine solution foaming at a continuous time point were randomly selected, and the first 8 data were listed as raw data, and the last 2 data were test data. The sensitivity of the model was verified. See Table 2 for details.

According to the modeling process, the raw data can be expressed as

$$
\begin{aligned}
x^{(0)} & =\left(x^{(0)}(1), x^{(0)}(2), x^{(0)}(3), \cdots, x^{(0)}(8)\right) \\
& =(8.9,10.4,11.2,7.4,16.3,8.8,26.7,11.6)
\end{aligned}
$$

According to accumulation of raw data, an accumulation sequence generated can be expressed as be expressed as

$$
\begin{aligned}
x^{(1)} & =\left(x^{(1)}(1), x^{(1)}(2), x^{(1)}(3), \cdots, x^{(1)}(8)\right) \\
& =(8.9,19.3,30.5,37.9,54.2,63,89.7,101.3)
\end{aligned}
$$

In the same way, according to the above process, the sum $b, y$ can be obtained,

$$
B=\left[\begin{array}{ll}
-14.1 & 1 \\
-24.9 & 1 \\
-34.2 & 1 \\
-46.1 & 1 \\
-58.6 & 1 \\
-75.9 & 1 \\
-95.5 & 1
\end{array}\right], \quad y_{n}=(10.4,11.2,7.4,16.3,8.8,26.7,11.6)^{\mathrm{T}}
$$

that is, $a=-0.024, u=2.462, \frac{u}{a}=-102.6$.

Therefore, the expression of the $\operatorname{GM}(1,1)$ model is

$$
x^{(1)}(i+1)=94.32 \mathrm{e}^{0.024 i}-102.6
$$

The average relative error can be calculated

$$
\Delta=\frac{1}{8} \sum_{i=1}^{8} \Delta_{i}=0.0347
$$

and the mean variance ratio is calculated.

$$
c=\frac{s_{1}}{s_{0}}=0.312
$$

As can be seen from Table 1, the test result is in first level, indicating that the model can be used for prediction.

Bringing the raw data of Table 2 into the Formula (12) gives the predicted value. See Table 3 and Figure 1. 


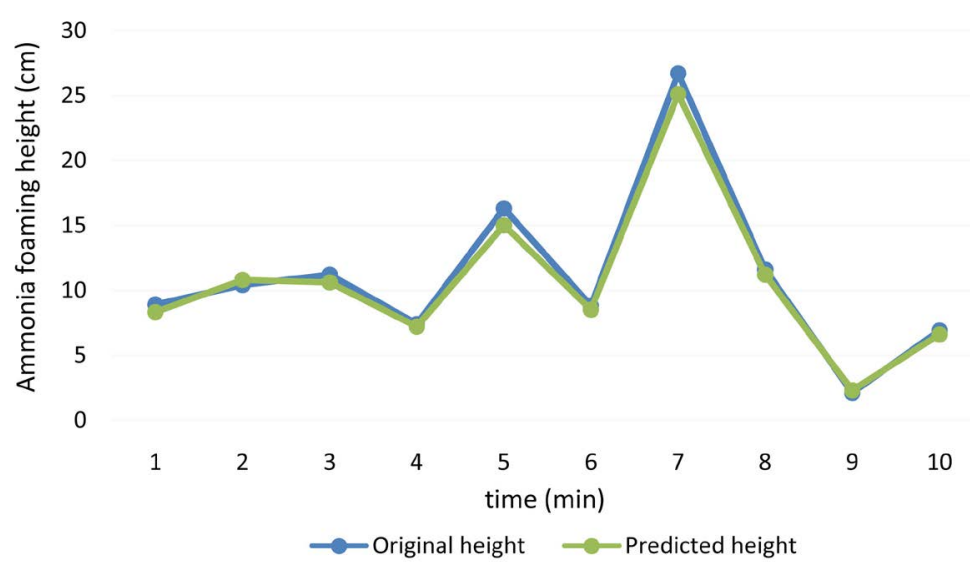

Figure 1. Contrast line analysis comparison chart.

Table 2. Statistics of foam height of amine solution.

\begin{tabular}{ccccccccccc}
\hline Time $(\mathrm{min})$ & 14 & 15 & 16 & 17 & 18 & 19 & 20 & 21 & 22 & 23 \\
\hline Height $(\mathrm{cm})$ & 8.9 & 10.4 & 11.2 & 7.4 & 16.3 & 8.8 & 26.7 & 11.6 & 2.1 & 6.9 \\
\hline
\end{tabular}

Table 3. Comparison of data before and after prediction.

\begin{tabular}{lcccccccccc}
\hline Original height $(\mathrm{cm})$ & 8.9 & 10.4 & 11.2 & 7.4 & 16.3 & 8.8 & 26.7 & 11.6 & 2.1 & 6.9 \\
\hline Predicted height $(\mathrm{cm})$ & 8.32 & 10.8 & 10.6 & 7.2 & 15 & 8.5 & 25.1 & 11.2 & 2.3 & 6.6 \\
\hline
\end{tabular}

Table 3 and Figure 1 show that the GM(1,1) gray model can be used to predict the foaming height of the amine solution, and the prediction precision is high, the method is simple, and it has a certain theoretical promotion value.

\section{Conclusions and Recommendations}

\subsection{Conclusion}

By using the $\operatorname{GM}(1,1)$ gray model to predict the foaming of the amine solution, the prediction results are close to the actual value, indicating that the prediction method has strong feasibility and operability. Because the foaming condition of the amine solution is affected by factors such as the condition of the equipment itself and human operation, it is worthy of further consideration and research to make accurate predictions.

\subsection{Suggestions}

1) Combine other methods to continuously optimize the $\mathrm{GM}(1,1)$ gray model to improve the prediction precision.

2) Conduct in-depth study of domestic and foreign literature, analyze and summarize factors influencing amine foaming from macro and micro perspectives, guarantee for accurate prediction.

\section{Acknowledgements}

This article was from the selected topic to finalized, Zhi-Xiong He professor to 
give the patient guidance and advice, express our heartfelt thanks to the teacher's pay.

\section{Conflicts of Interest}

The author declares no conflicts of interest regarding the publication of this paper.

\section{References}

[1] Hui, X., Feng, Y.S., Liu, P.H. and Wang, J.F. (2014) Cause Analysis and Control Measures of Amine Solution Foaming. GuangZhou Chemical Industry, 42, 188-190.

[2] Guo, L.C. and Wang, L.J. (2014) Analysis and Countermeasures of Amine Solution Foaming in Gas Desulfurization Equipment. Technology and Development of Chemical Industry, 43, 66-67.

[3] Huang, T.T. and Xie, Y.Z. (2017) Analysis of Foaming Problem of Natural Gas MDEA Desulfurization Process Solution. Refining \& Chemical Industry, 28, 20-21.

[4] Zhang, G.J. (2017) Study on Foaming Characteristics of Natural Gas Decarbonated Amine Solution. China University of Petroleum (East China), Shanghai.

[5] Liu, S.F. (2010) Grey System Theory and Its Application. Science Press, Beijing, 32-33. 